Background Sleep is important to elite athletes as it plays a key role in repair of cellular damage. In recent years, there has been increasing interest in the role of sleep in athletes.

Objectives The aim of this study is to give a systematic overview of what is known about the impact of sleep on sport injuries and injury recovery. The following questions will be addressed: 1. What is the prevalence of sleep disturbances in athletes? 2. What is the impact of sleep on injury risk? 3. What is the relationship between sleep and recovery after training and/or injury? 4. What is the influence of sleep and nutrition on recovery?

Design Systematic review

Methods Screening of PubMed, Web of Science, Cinahl and Cochrane Library on the keywords 'athletic injuries', 'football', 'soccer', 'sleep deprivation', 'fatigue', 'circadian rhythm', 'insomnia', 'rehabilitation', 'recovery', 'prevention' between January 1, 2002 and November 14, 2020; systematic review after assessment for methodological quality.

Results There is strong evidence that athletes in general are at high risk for a poor sleep duration and quality. In contrast to the expectations, most athletes $(>85 \%)$ are mid-range to morning types and tend to pursue and excel in sports that match their chronotype.

Reduced sleep $(<8 \mathrm{~h})$ is related with 1.7 more risk of having an injury and a negative effect on recovery after training. There is an increased proinflammatory state, which affects a person's resistance to a simple infectious disease, and may affect an athlete's training capacity and potential.

Sleep is also associated with recovery from injuries. Studies found that sleep affected concussion recovery time and an increased risk of a re-injury during rehabilitation.

Conclusion The importance of sleep remains a main issue in competitive sporters. Training and competition schedules should take sleep into account in order to avoid injuries and prolonged rehabilitation after injury.

\section{THE ASSOCIATION BETWEEN COVID-19 AND MUSCLE STRAIN INJURY OCCURRENCE IN ELITE SOCCER PLAYERS: A PROSPECTIVE STUDY}

${ }^{1}$ Evi Wezenbeek, 'Sander Denolf, 'Dries Pieters, ${ }^{1,2}$ Jan Bourgois, ${ }^{3}$ Renaat Philippaerts, ${ }^{4}$ Bram De Winne, 'Joke Schuermans, 'Steven Verstockt, 'Erik Witvrouw. 'Ghent University, Ghent, Belgium; ${ }^{2} K A A$ Ghent, Ghent, Belgium; ${ }^{3}$ Royal Standard de Liège, Liège, Belgium; ${ }^{4}$ Zulte Waregem, Waregem, Belgium

\subsection{6/bjsports-2021-IOC.418}

Background Because of the high injury rate currently seen in soccer players and the exceptional pandemic circumstances, the question arises to what extent this reported increase in injury incidence is associated with COVID-19.

Objectives The aim of this study was to investigate the association between COVID-19 and muscle strain injury occurrence in elite athletes.

Design A prospective cohort study

Setting During the first half of the 2020-2021 season, injury data was collected by using the UEFA injury card. Assessment of SARS-CoV-2 infection was performed by means of a polymerase chain reaction (PCR) test before each official game.
Patients (or Participants) Three Belgian professional male soccer teams (84 players in total) participated.

Interventions (or Assessment of Risk Factors) Regression analysis was performed to identify the risk of developing a muscle strain injury after SARS-CoV-2 infection.

Main Outcome Measurements Muscle strain injury, COVID-19 diagnosis, quarantine duration.

Results Eighteen players developed a muscle strain injury during the study period (June 2020- January 2021), of which 11 players were diagnosed with COVID-19. Regression analysis showed a significant association between SARS-CoV-2 infection and the development of muscle strain injuries $(p=0.010)$. The athletes that developed a muscle strain injury after COVID-19 diagnosis were all injured within the first month (15.71 \pm 11.74 days) after sports resumption and showed a longer quarantine duration (14.57 \pm 6.50 days) compared to the SARS-CoV-2 infected players that did not develop a muscle strain injury (11.18 \pm 5.25 days).

Conclusion This study demonstrated a five-time higher risk to develop a muscle strain injury after a SARS-CoV-2 infection in elite soccer players. Although this should be examined further, it seems possible that short-term detraining effects due to quarantine are associated with a higher risk of muscle strain injury and could possibly be related to lower physical readiness and higher rates of fatigue.

\section{SAFETY AND RISK AWARENESS FOR HEALTH AND WELLBEING EVALUATION IN ELITE RUGBY: A QUANTITATIVE SURVEY DEVELOPMENT}

${ }^{1}$ Yanbing Chen, ${ }^{2}$ Conor Buggy, 'Seamus Kelly. 'Institute of Sport and Health, School of Public Health, Physiotherapy and Sports Science, University College Dublin, Dublin, Ireland; ${ }^{2}$ Centre for Safety and Health at Work, School of Public Health, Physiotherapy and Sports Science, University College Dublin, Dublin, Ireland

\subsection{6/bjsports-2021-IOC.419}

Background Despite the physical, competitive nature of elite athletes and unique features of sport culture, no Occupational Safety and Health (OSH) related measurement instruments exist in sport.

Objective As a part of a wider project focusing on health and safety awareness in elite sport, the purpose of this study was to develop a survey instrument for the evaluation of risk and safety awareness among elite rugby players.

Design Based on an established conceptual framework incorporating OSH theories, the survey was developed by adopting questions from existing validated questionnaires through an iterative process, with the feedback from a multidisciplinary team of experts and pilot test.

Setting Elite division rugby (union) team in Ireland

Participants Players from an elite rugby team in Ireland $(n=50)$ participated the advanced pilot test.

Assessment of Risk Factors The key themes include current health outlook, tackle behaviour, awareness of risk acceptance, reasons for risk-taking, and safety consideration for other players.

Main Outcome Measurements The data were imported to SPSS for analysis of survey reliability and validity, such as the adoption of the Kaiser-Meyer-Olkin (KMO) measure. 